\title{
Warum ist die Regeneration kernloser Protoplasma- stücke unmöglich oder erschwert?
}

\author{
Von \\ Jacques Loeb.
}

(From the Hull Physiological Laboratory of the University of Chicago.)

Eingegangen am 30. März 1889.

Es ist durch eine Reihe von Versuchen sichergestellt, dass Sauerstoffzufuhr für die Entwickelung von Eiern sowie für Vorgänge der Regeneration nöthig ist. Der Grund hierfür dürfte unter Anderem darin zu suchen sein, dass, wie ich in zwei frihheren Aufsätzen ${ }^{1}$ ) ausgefiuhrt habe, synthetische Processe für diese Vorgänge nöthig sind, und dass die Synthesen an die Sanerstoffzufuhr gebunden sind. Es ist nun bekannt, dass, wenn man eine Zelle in mehrere Stücke schneidet, nur die kernhaltigen Stiicke regenerationsfähig sind, während die kernlosen Stiucke bald zu Grunde gehen. Man hat das so gedeutet, dass der Kern spceifische organbildende Stoffe enthalte, die er an das Protoplasma abgebe. Dieser Schluss ist aber nicht bindend. Es könnte auch möglich sein, dass der Kern nur für das Zustandekommen der Oxydationsvorgänge nöthig sei. Mit dem Ausfallen des Kerns wïrde dann anch eine Hemmung oder Herabsetzung der Oxydationsvorgänge verknüpft sein. Das müsste genügen, um das Zustandekommen der Regeneration kernloser Zellstiicke zu verhindern. Ich will nun prüfen, in wie weit das vorliegende Thatsachenmaterial eine solche Annahme stützt.

Für das Zustandekommen der Oxydationen im lebenden Gewebe ist die Anwesenheit von katalytisch wirkenden Substanzen nöthig, welche entweder den atmosphärischen Sauerstoff »aktiviren * oder die Verbindungen in den Zellen geeignet machen, den atmosphärischen Sauerstoff energischer aufzunehmen. Die Mehrzahl der Forscher

1) Assimilation and Heredity. The Monist. 1898, und: Über den Einfluss von Säuren und Alkalien anf embryonale Entwickelung und Wachsthum. Archiv f. Entwickelungsmech. Bd. VII. 1898. 
nimmt an, dass der Sauerstoff in den Geweben aktivirt wird und dass hierbei bestimmte Stoffe katalytisch thätig sind, welche wir nach Traube "Sauerstoffüberträger" nennen wollen. Es ist nun gelungen, diese Sauerstoffüberträger aus den Zellen zu extrahiren und man hat zeigen können, dass in diesem Zustand die Sauerstoffüberträger noch im Stande sind, diejenigen Oxydationen auszuführen, welche für die lebende Substanz charakteristisch sind.

Die werthvollste Entdeckung hat auf diesem Gebiete unstreitig SPITZER gemacht ${ }^{1}$ ). Er hat nämlich festgestellt, dass die in Gewebsextrakten enthaltenen Substanzen, welche dic Sauerstoffübertragung begiunstigen (Oxydationsfermente), zur Gruppe der Nucleoproteide gehören. Die Nucleoproteide sind typische Kernstoffe. Alle diese Nucleoproteide enthielten Eisen. Wir wissen, dass gerade Eisensalze geeignet sind, katalytisch die Oxydationen zu befördern. Es ist kein Grund vorhanden, zu bezweifeln, dass, was für die wïsserigen Extrakte der Zellen gilt, auch für Nucleoproteide der lebenden Zellen gilt. MaCALLum hat in der Chromatinsubstanz der Zellkerne Eisen nachgewiesen. Die Arbeiten von SprTzer machen es also wahrscheinlich, dass der Kern das Oxydationsorgan der lebenden Substanz ist.

Wir wollen nun zusehen, ob das Verhalten kernloser Zellsticke dieser Voraussetzung entspricht. VERwons schildert die Veränderungen der Psendopodien in diesen Fällen folgendermaßen. In den Psendopodien eines kernlosen Stiuckes von Orbitolites z. B. bilden sich Trüpfehen, die zum Theil zu größeren Tropfen zusammenfließen. Die Verbindung zwischen den einzelnen T'Tropfen reißt ab. "Schließlich, wach ca. 5-7 Stunden, bildet die Centralmasse einen rundlichen Klumpeu ohue Pseudopodien, um den eine Anzahl von kleineren und größeren Protoplasmakugeln, Tropfen und Spindeln umher zerstreut liegen ${ }^{2}$ ). Verworn hat im Anschluss an Berthold eine Theorie der amöboiden Bewegung aufgestellt, nach der das Ausstrecken und Einziehen von Pseudopodien auf Ändenungen der Oberflichenspannung zuriickgefïhrt wird. Voraussetzung dieser Theorie ist der fliissige Aggregatznstand der Pseudopodien ${ }^{3}$ ). Diese Voraussetzung ist eine physikalische Unmöglichkeit.

1) Die Bedeutung gewisser Nucleoproteide fïr die oxydative Leistung der Zelle. PruÜGer's Archiv. Bd. 67. 1 S97.

2) Verworn, Die physiologische Bedeutung des Zellkerns. PrLüger's Archiv. Bd. 51. 1892.

3) Die Bewegung der lebendigen Substanz. Jena 1892. pag. 38. 
Die Pseudopodien von Orbitolites sind nach der Zeichnung von Verworn Cylinder, deren Höhe wohl hundertmal größer als ihr Umfang ist. Solche Flüssigkeitseylinder können nicht bestehen. Es hat seinen guten Grund, dass der Regen in Tropfen fällt und nicht in Strahlen. Ist $r$ der Radius, $h$ die Höhe eines flüssigen Cylinders, so kann derselbe nur so lange existiren, als $h \leqq 2 r \pi$ ist. Aus diesem Grunde allein ist die ganze Verwors'sche Kontraktionstheorie falsch, worauf ich aber hier nicht näher eingehen will 1). Aus diesen Thatsachen der Oberflächenspannung folgt, dass die Pseudopodien der Rhizopoden nicht flüssig sein können, sondern ein festes Gerïst oder eine feste Membran besitzen müssen. Sobald dieses feste Gerüst oder die feste Membran sich verflïssigt, muss das Pseudopodium den Gesetzen der Oberflächenspannung folgen und in Tropfen zerfallen. Das Letztere ist nun meines Erachtens der Vorgang, der in kernlosen Orbitolitesstïcken stattfindet.

Ist diese Verflüssigung fester Bestandtheile ein Vorgang, der auf Sauerstoffmangel hindeutet? Das ist zweifellos der Fall. Ich habe vor 4 Jahren nachgewiesen, dass die Zellwände der Furchungszellen von Ctenolabrus sich verflüssigen, wenn man ihnen den Sauerstoff entzieht. Lässt man den Sauerstoff dann wieder zuströmen, so tritt von Neuem eine Bildung von Zellwänden ein ${ }^{2}$ ). BUDGETT hat in meinem Laboratorium den Nachweis geführt, dass Sauerstoffentziehung auch bei Infusorien zur Auflösung von Zellwänden führt und dass gewisse Gifte ebenso wirken ${ }^{3}$ ). KÜHNe hat dieselbe Thatsache bei Sauerstoffmangel beobachtet ${ }^{4}$ ). Die Verflüssigung der Pseudopodien bei Entfernung des Zellkerns, die Unfähigkeit kernloser Infusorienstïcke eine neue Cuticula zu bilden, entsprechen ganz der Annahme, dass kernlose Zellsticke sich im Zustande verringerter Oxydationsthätigkeit befinden.

Ich habe diese Anschauung schon seit mehreren Jahren in meinen Vorlesungen vertreten und ich trug mich mit dem Gedanken, Versuche mit höherem Sauerstoffdruck an entkernten Infusorien anzustellen. Wenn meine Theorie richtig wäre, so miisste es möglich

1) Die chemotropischen Vorstellungen VerworN's liasse ich unerwähnt, weil sie jeder thatsächlichen Grundlage entbehren.

2) Untersuchungen iuber die physiologischen Wirkungen des Sauerstoffmangels. PFuÜGer's Archiv. Bd. 62. 1895.

3) On the Similarity of Structural Changes Produced by Lack of Oxygen and Certain Poisons. The American Journal of Physiology. Vol. I. 1898.

4) Über die Bedeutung des Sanerstoffs für die vitale Bewegung Zeitschr. f. Biologie. Bd. 36. pag. 472, 1898. 
sein, durch bessere Sauerstoffzufuhr das Leben kernloser Zellstiicke zu verlängern. Allein es scheint mir, dass dieser Versuch schon ausgeführt ist. Wïhrend nämlich Nussbaum, Gruber und Verworn fanden, dass kernlose Infusorienstïcke schon nach 2 Tagen zu Grunde gehen und nur ganz ausnahmsweise einige Tage länger leben, scheinen alle Botaniker, welche denselben Versuch an chlorophyllhaltigen Zellen (z. B. Algen) ausführten, gefunden zu haben, dass kernlose Zelisticke 5-6 Wochen am Leben bleiben $\left.{ }^{1}\right) !$ In solchen Zellstiucken fand Assimilation statt. Mir scheint es nun, als ob die ungleich längere Lebensdauer kernloser Algenstücke von einer großen Bedeutung für die Beurtheilung der Funktion des Zellkerns sei. Bei der Assimilation der Kohlensäure wird bekanntlich Sauerstoff frei. Im Lichte sind desshalb kernlose Stücke von Algen in einem Znstande günstiger Sauerstoffversorgung im Vergleiche mit chlorophyllfreien kernlosen Infusorienfragmenten.

Es scheint mir desshalb, dass alle einstweilen bekannten Thatsachen sich natürlich der Vorstellung unterordnen, dass der Kern das Oxydationsorgan der lebenden Substanz sei; und dass kernlose Zellstücke nur desshalb nicht im Stande sind, zu regeneriren, weil in ihnen die Oxydationsthätigkeit auf ein zu geringes Maß heruntergesunken ist. Derartige Stucke gehen langsam an Erstickung zu Grunde.

Die Frage nach der Bedeutung des Zellkerns für die Entwickelung und Vererbung tritt, wenn diese Ansichten richtig sind, in ein anderes Licht. Es geht auch nicht länger an, den lebenden Organismus als einen Verband einzelner Zellen anzusehen. "Unter dem zelligen Aufbau verstehen wir den Umstand, dass es eine bestimmte, aber für verschiedene Formen und Gewebe variirende maximale Distanz des Protoplasmaelementes vom nächsten Kern geben muss ${ }^{2}$ ). Die Ursache hierfür können wir jetzt verstehen: Wird die Entfernung zu groß, so geht das betreffende Protoplasmaelement an Erstickung zu Grunde. Dass außerdem ein Stoffaustausch zwischen Protoplasma und Kern stattfindet, will ich natürlich nicht in $\mathbf{A b}$ rede stellen; es ist möglich oder wahrscheinlich, dass mit der oxydativen Thätigkeit die Bedeutung des Zellkerns nicht erschöpft ist. Es ist anch kaum nöthig noch besonders darauf hinzuweisen, dass

1) Klebs, Über den Einfluss des Kerns in der Zelle. Biologisches Centralblatt. Bd. VII. 1888.

2) LOEB, Einige Bemerkungen ïber Begriff, Geschichte und Litteratur der allgemeinen Physiologie. PfLüGER's Archiv. Bd. 69. 1897. 
ich nicht der Meinung bin, dass ohne Kern alle Oxydationsvorgänge im Protoplasma aufhören; es geht vielmehr aus allen Thatsachen hervor, dass sie nur erheblich verringert sind.

Chicago, 15. März 1899.

Nachtrag bei der Korrektur.

O. Scruurze 1 ) scheint meine Arbeit »Über die physiologisehe Wirkung des Sauerstoffmangels « in einem wesentlichen Punkte missverstanden zu haben. Er behauptet nämlich Folgendes: „J. LоEB stellte auf Grund seiner experimentellen Arbeiten an dem sich furchenden Knochenfisch Ctenolabrus und Fundulus den Satz auf, dass die Eier des ersteren zur Furchung ebenso des $O$ benöthigten, wie die Eier des letzteren ihn völlig entbehren könnten." DEMook hatte behauptet, dass ohne Sauerstoff keine Furchung mehr möglich sei. Gegen diese zu weit gehende Behauptung wendete ich mich, indem ich zeigte, dass dem gleichen Sauerstoffmangel gegeniiber ganz nahestehende Formen sich sehr verschieden verhalten können. Während Ctenolabrus-Eier sich der Behauptung Demoor's nahezu fügen, geht unter den gleichen Umständen die Furchung bei Fundulus 12 Stunden oder länger weiter. Dass ohne Sauerstoff die Furchung schlieBlich auch bei Fundulus zum Stillstand kommt, habe ich ja in derselben Arbeit gezeigt. Denselben Unterschied wie in Bezug auf die Furchung fand ich auch für die Herzthätigkeit der beiden Formen. Das Herz des Ctenolabrus-Embryo steht bald still, wenn man ihm den Sauerstoff entzieht, während das Her\% des Fundulus-Embryo unter diesen Umständen 12 Stunden weiter schlägt. Ich habe aber nie geglaubt oder behauptet, dass das Fundulus-Herz den Sauerstoff fuir immer entbehren könne. Wie sich in Bezug auf die Bewegung Übergänge finden zwischen strikten Aeroben und strikten Anaeroben, so glaube ich, dass das Gleiche auch für Zelltheilung und Wachsthum der Fall ist. - In ScHuL'rze's Versuchen handelt es sich ubbrigens nicht bloß um Sauerstoffmangel, sondern auch um Kohlensäurevergiftung, was ihm natiurlich nicht entgangen ist. Dagegen scheint es mir, dass er die Kohlensäurewirkung in seinen Versuchen unterschätzt. Ich halte es für dringend nöthig, dass man die Kohlensäure beseitigt, wenn man die Wirking des Sanerstoffmangels untersuchen resp. beurtheilen will.

1) O. Schultze, Über den Einflnss des Luftmangels auf die erste Entwickelung des Eies. Verh. d. physik.-med. Ges. Wiirzburg. 1899. 